\title{
Dual Nanopatterns Consisting of Both Nanodots and Nanoholes on a Single Substrate
}

Chungryong Choi, Myeongcheol Go, So Yeong Park, Sukwon Kang, Yeseong Seo, Jaeyong Lee, Jin Kon Kim*

National Creative Research Center for Smart Block Copolymer Self-Assembly, Departments of Chemical Engineering, Pohang University of Science and Technology, 77 Cheongam-Ro, NamGu, Pohang, Kyeongbuk, 37673, Korea,

E-mail : jkkim@postech.ac.kr 


\section{Synthesis of PS-hv-PMMA (3)}

5-propargylether-2-nitrobenzyl bromoisobutyrate [Alkyne-ONB-ATPR (1)] was synthesized according to the scheme in the Figure S1(a). ${ }^{1}$ Molecule 1 contains $o$-nitrobenzyl alcohol (ONB) photo cleavable linker, alkyne group for alkyne-azide click reaction, and ATRP initiator for the polymerization of methylmethacrylate. Figures $\mathrm{S} 1(\mathrm{~b}-\mathrm{e})$ show ${ }^{1} \mathrm{H}$ and ${ }^{13} \mathrm{C}$ NMR spectra of purified Alkyne-ONB and 1, from which both were successfully synthesized.

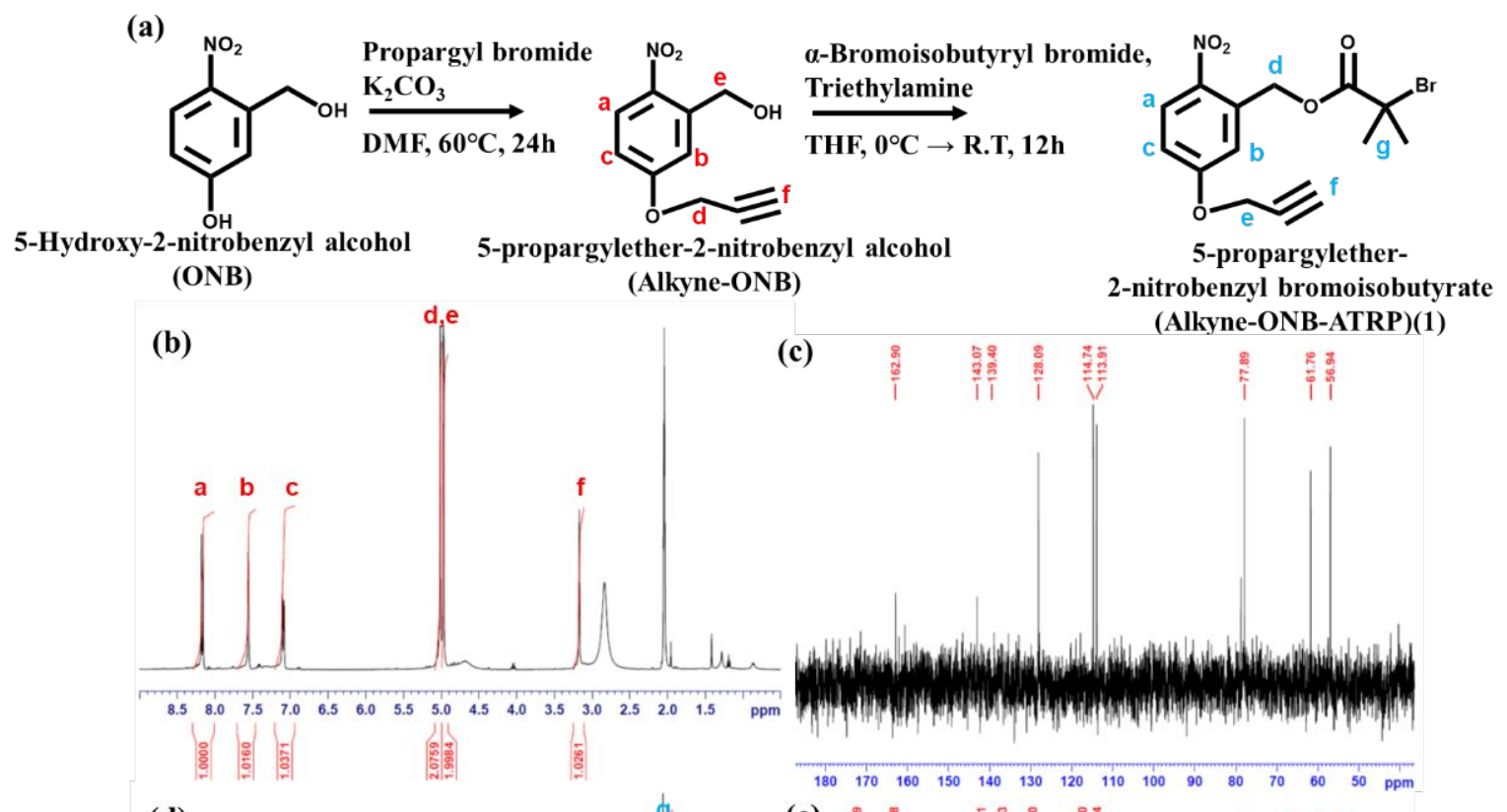

(d)

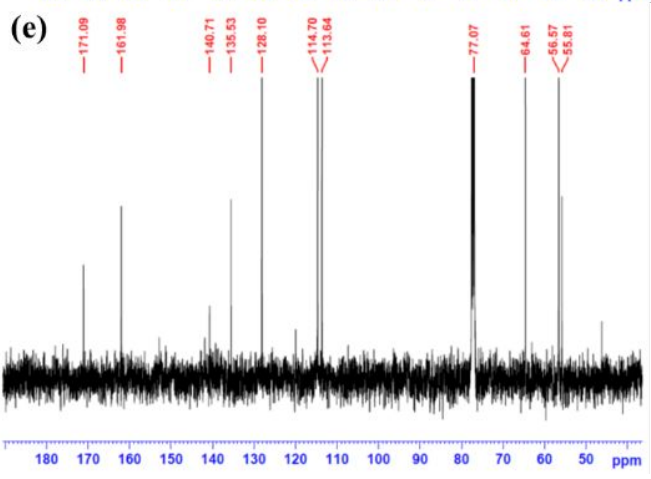

Figure S1. (a) Synthetic scheme of Alkyne-ONB-ATRP (1). With ONB photocleavable linker, alkyne group, and ATRP initiator. (b) ${ }^{1} \mathrm{H}$ NMR and (c) ${ }^{13} \mathrm{C}$ NMR for Alkyne-ONB. (d) ${ }^{1} \mathrm{H}$ NMR and (e) ${ }^{13}$ C NMR for Alkyne-ONB-ATRP (1). 
End functionalized PS with azide group (PS-N $\mathrm{N}_{3}$ ) was synthesized according to the scheme in Figure S2a. First, we polymerized PS by ATRP with an initiator of (1-bromoethyl) benzene. The initiator (20 ul), styrene monomer (40 ml), Copper(I) bromide (CuBr) $(60 \mathrm{mg}), \mathrm{N}, \mathrm{N}, \mathrm{N}^{\prime}, \mathrm{N}^{\prime \prime}, \mathrm{N}^{\prime \prime}-$ pentamethyldiethylenetriamine (PMDETA, 138 $\mu$ ) were put into a reactor and conducted by freeze-thaw three three times to remove air. The reactor was stirred in oil bath at $90{ }^{\circ} \mathrm{C}$. The molecular weight was controlled by reaction time. After reaction, the reacted solution passed through an alumina column and was precipitated in methanol to remove the catalysts. Next, to substitute $\mathrm{Br}$ end group with $\mathrm{N}_{3}, \mathrm{PS}-\mathrm{Br}$ and an excess amount of $\mathrm{NaN}_{3}$ were stirred in DMF under nitrogen atmosphere for $12 \mathrm{~h}$ at $80^{\circ} \mathrm{C}$. After the reaction, DMF was removed in rotary evaporator and the product was dissolved in THF. Then the solution was passed through as alumina column to remove unreacted $\mathrm{NaN}_{3}$ and final product was obtained by precipitating in methanol. The product gives a single peak of SEC trace with narrow PDI (1.15), as shown in Figure S2(b).

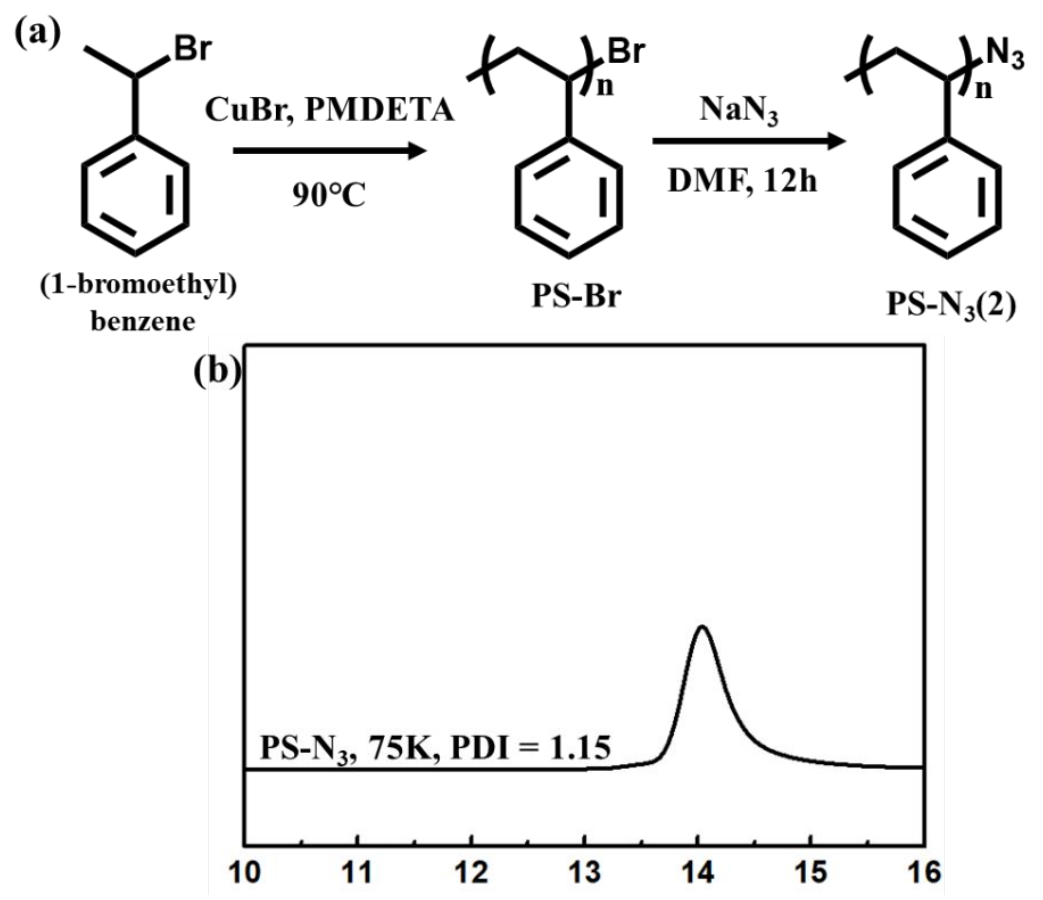

Figure S2. (a) Synthetic scheme of PS-N $\mathrm{N}_{3}$ and (b) SEC trace of synthesized PS-N $\mathrm{N}_{3}$. 
Finally, PS- $h v$-PMMA(3) was synthesized by one-pot reaction of ATRP and azide-alkyne click reaction. Since ATRP and click reaction require the same catalysts, $\mathrm{CuBr}$ and PMDETA, it was possible to simultaneously synthesize PS- $h v$-PMMA in a single reactor, although the yield was smaller compared to that obtained by the separate click reaction and ATRP. Molecule $1(0.05 \mathrm{~g})$, molecule $2(0.3 \mathrm{~g}), \mathrm{CuBr}(6 \mathrm{mg})$, PMDETA $(12 \mu \mathrm{l})$ were added in the mixture of MMA (10 ml) and anisole $(6 \mathrm{ml})$. Freeze-thaw was conducted three times and the reactor was stirred in an oil bath at $90{ }^{\circ} \mathrm{C}$ for $20 \mathrm{~min}$. After the reaction, the solution passed through an alumina column to remove $\mathrm{CuBr}$. To remove unreacted PMMA homopolymers, the solution was precipitated in methanol/acetic acid solution. The reaction did not proceed $100 \%$ even though an excess amount of PS was used for the click reaction. Thus, the product was precipitated in a mixture of cyclohexane $/ n$-hexane to remove unreacted PS-N N $_{3}$.The synthesized PS- $h v$-PMMA was characterized by SEC, as shown in Figure 1, and the number average molecular weights of PS and PMMA blocks in PS- $h v$-PMMA were 75,000 and 25,000g/mol, respectively (Table 1), and narrow PDI of 1.16. 


\section{Bulk morphology of PS- $h v$-PMMA}

Figure S3 gives TEM image and SAXS profile PS- $h v$-PMMA in bulk. Since PS blocks are selectively stained by $\mathrm{RuO}_{4}$, PS microdomains become dark. Thus, white PMMA cylinder microdomains are clearly seen. Also, from the SAXS profile with characteristic peaks at the positions of $\mathrm{q}^{*}: \sqrt{3} \mathrm{q}^{*}: \sqrt{7} \mathrm{q}^{*}\left(\mathrm{q}^{*}\right.$ is the the first peak position), PS- $h v$-PMMA shows cylindrical nanodomains in bulk. The domain spacing $\mathrm{L}_{0}\left(=2 \pi / \mathrm{q}^{*}\right)$ was $46 \mathrm{~nm}$.
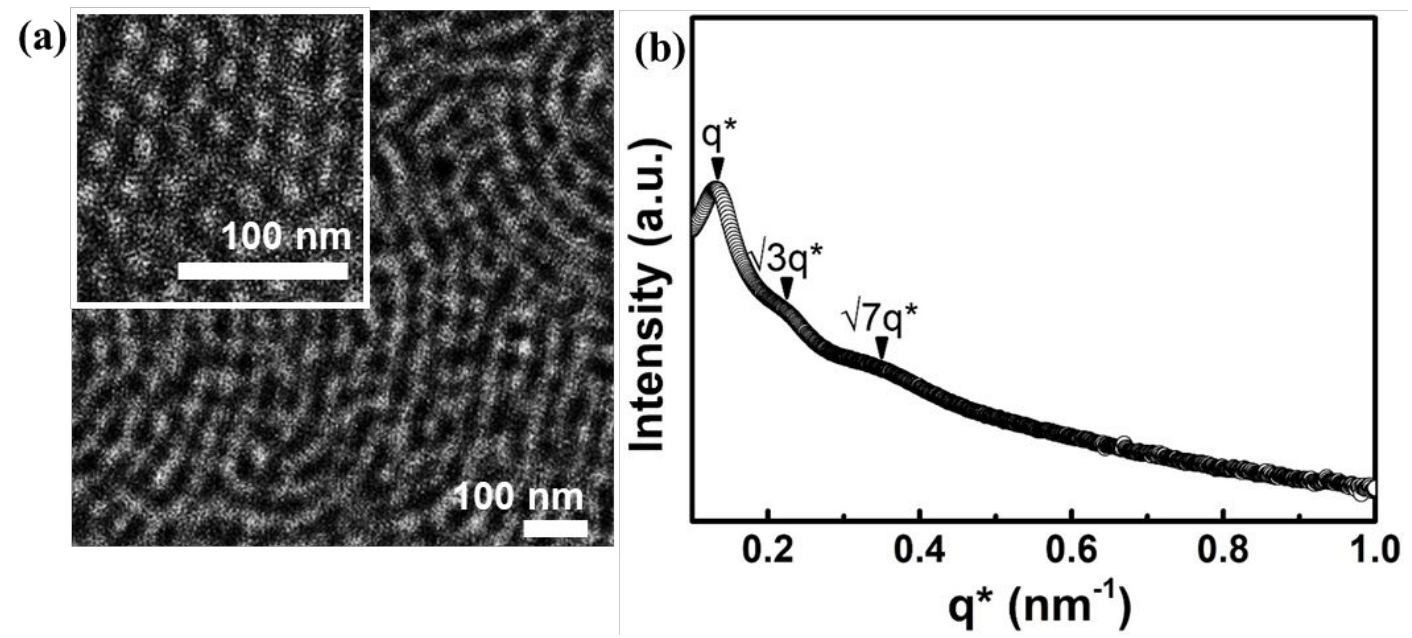

Figure S3. (a) TEM image stained by $\mathrm{RuO}_{4}$ and (b) SAXS profile of PS- $h v$-PMMA in bulk. 


\section{Thin film morphology of PS- $h v$-PMMA}

When PS- $h v$-PMMA thin film was spin-coated on a neutralized silicon substrate with a thickness of $46 \mathrm{~nm}\left(\sim 1 \mathrm{~L}_{0}\right)$, vertically oriented PMMA cylinders were obtained, confirmed by AFM image as shown in Figure S4a. Figures S4b and c give the GISAXS pattern and in-plane scattering profile. The peaks observed at $\sqrt{3} \mathrm{q}^{*}$ and $\sqrt{7} \mathrm{q}^{*}$ indicate hexagonally packed cylinders. Also, the streak as shown in GISAXS pattern along the $\mathbf{q}_{\mathrm{z}}$ direction indicates that the cylindrical nanodomains are vertically oriented to the substrate.

(a)

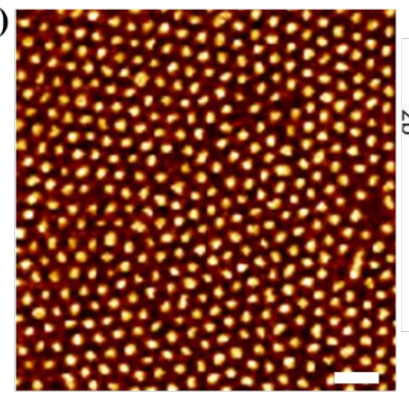

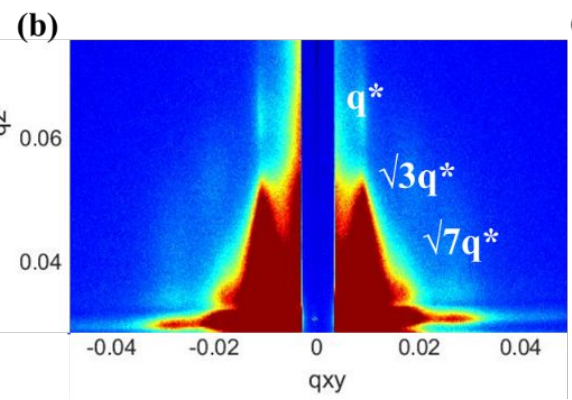

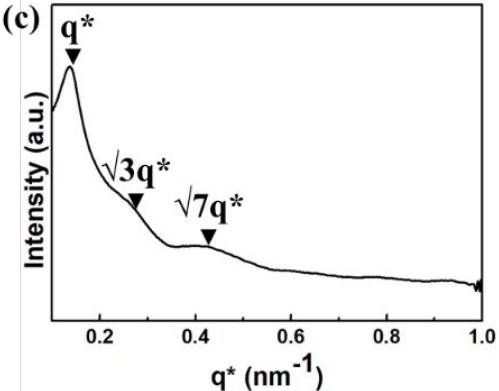

Figure S4. (a) Phase contrast AFM image (Scale bar is $100 \mathrm{~nm}$ ), (b) GISAXS pattern and (c) the in plane scan $\left(\mathbf{q}_{\mathrm{xy}}\right)$ of GISAXS pattern of PS- $h v$-PMMA thin film with $46 \mathrm{~nm}$ thickness on a neutralized silicon substrate. 


\section{FT-IR spectra}

Since nanoholes are fabricated by complete removal of PMMA block by $\mathrm{O}_{2}$ RIE, thin films should have only PS chains. On the other hand, thin films with nanodots should have only PMMA chains. Figure S5 gives FT-IR spectra of thin film with nanoholes and nanodots, respectively. Thin film with nanoholes do not show the characteristic peaks corresponding to PMMA chains (for instance, 1750 and $1110 \mathrm{~cm}^{-1}$ for $\mathrm{C}=\mathrm{O}$ and $\mathrm{C}-\mathrm{O}$ stretch), while no characteristic peaks belonging PS chains (1500 and $750 \mathrm{~cm}^{-1}$ for aromatic ring) appeared in another thin film with nanodots, as shown in figure S5.

(a)

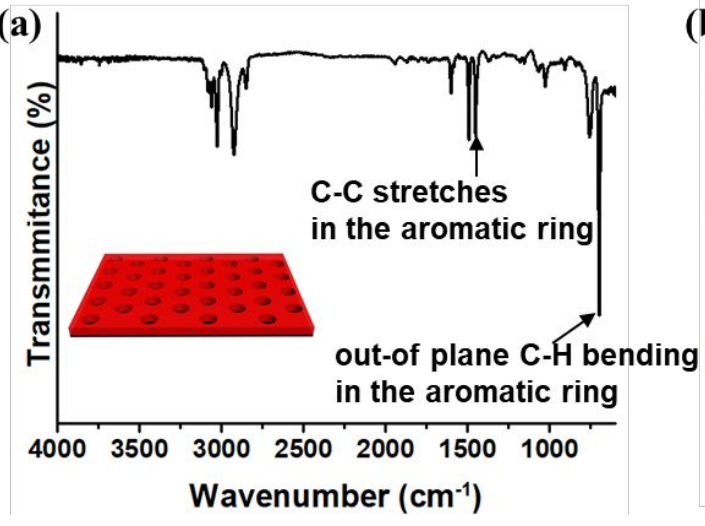

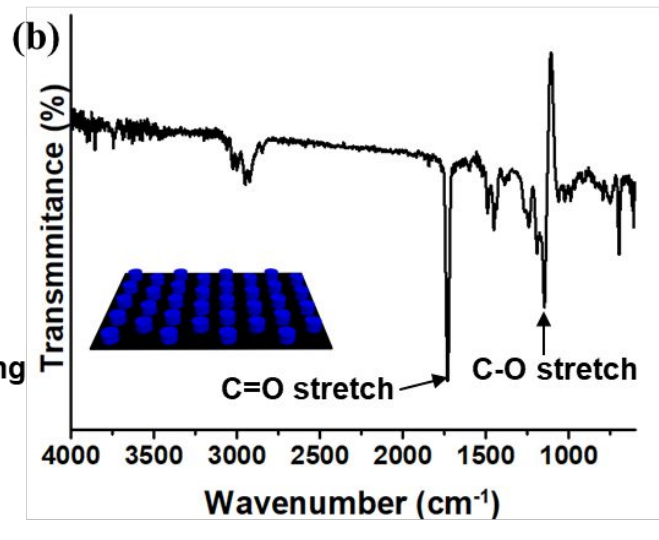

Figure S5. FT-IR spectra of thin film with (a) nanoholes and (b) nanodots.

\section{References}

1. Schumers, J. M.; Gohy, J. F.; Fustin, C. A., A versatile strategy for the synthesis of block copolymers bearing a photocleavable junction. Polym. Chem. 2010, 1 (2), 161-163. 